02

\title{
Исследование влияния ионной проводимости на коэффициент оптического поглощения кристаллов трибората лития при воздействии высокоинтенсивного непрерывного лазерного излучения
}

\author{
(C) И.В. Грищенко ${ }^{1}$, Ю.С. Стирманов ${ }^{2}$, А.В. Коняшкин ${ }^{2}$, О.А. Рябушкин ${ }^{2}$ \\ ${ }^{1}$ Московский фризико-технический институт (Национальный исследовательский университет), \\ 141700 Долгопрудный, Московская обл., Россия \\ ${ }^{2}$ Фрязинский фрилиал Института радиотехники и электроники им. В.А. Котельникова РАН, \\ 141190 Фрязино, Московская обл., Россия \\ e-mail: ivan.grishchenko@phystech.edu
}

Поступила в редакцию 15.03.2020 г.

В окончательной редакции 15.03.2020 г.

Принята к публикации 20.05.2020 г.

\begin{abstract}
Представлены результаты исследования влияния ионной проводимости кристалла трибората лития на коэффициент оптического поглощения при воздействии лазерного излучения с высоким уровнем непрерывной мощности на длине волны $1070 \mathrm{~nm}$.
\end{abstract}

Ключевые слова: триборат лития, пьезорезонансная лазерная калориметрия, оптическое поглощение, лазерное излучение, ионная проводимость, эквивалентная температура.

DOI: $10.21883 /$ OS.2020.09.49862.95-20

\section{Введение}

Эффективность преобразования частоты лазерного излучения в нелинейно-оптических кристаллах зависит от согласования фаз взаимодействующих волн, которое в случае генерации второй гармоники с первым типом взаимодействия определяется равенством показателей преломления. Нарушение его происходит, в частности, при изменении средней температуры и возникновении продольных и поперечных температурных градиентов в кристалле. Как правило, в рабочем диапазоне длин волн лазерного излучения нелинейно-оптические кристаллы имеют небольшие коэффициенты поглощения. Однако в случае преобразования высоких мощностей лазерного излучения даже небольшое оптическое поглощение кристаллов может приводить к значительному неоднородному разогреву и, как следствие, к снижению эффективности преобразования. В настоящее время мощность волоконных одномодовых иттербиевых лазеров достигает $20 \mathrm{~kW}$ в непрерывном режиме генерации [1]. При воздействии на кристаллы лазерного излучения киловаттного уровня мощности возможно образование локальных дефектов, деградация и даже оптическое разрушение кристаллов. Образование локальных микродефектов предшествует оптическому разрушению кристалла. Поэтому задетектировав формирование микродефектов, можно определить припороговые значения интенсивности, при которых происходит разрушения кристалла.

В настоящее время для преобразования лазерного излучения высокой интенсивности широко используются нелинейно-оптические кристаллы группы боратов $\beta$ -
$\mathrm{BaB}_{2} \mathrm{O}_{4}$ (BBO) и $\mathrm{LiB}_{3} \mathrm{O}_{5}$ ( $\left.\mathrm{LBO}\right)$, которые были синтезированы в 1980-хгг. [2,3]. Несмотря на сравнительно небольшие коэффициенты квадратичной нелинейности $\left(d_{22}=2.2 \cdot 10^{-12} \mathrm{~m} / \mathrm{V}\right.$ у ВВО и $d_{32}=0.85 \cdot 10^{-12} \mathrm{~m} / \mathrm{V}$ у LBO), они имеют очень высокую лучевую прочность [4]. Кроме того, край фундаментального оптического поглощения данных кристаллов сильно смещен в УФ область спектра (кристаллы LBO и BBO прозрачны в диапазонах от 0.16 до $2.3 \mu \mathrm{m}$ и от 0.2 до $2.6 \mu \mathrm{m}$ соответственно) [4].

Объектом исследования в настоящей работе являлся кристалл LBO, который широко применяется для генерации гармоник мощного лазерного излучения в видимой и УФ частях спектра. Кристаллы LBO отличаются низким линейным коэффициентом оптического поглощения [4,5] и высоким порогом оптического разрушения (для излучения с длиной волны $1.064 \mu \mathrm{m}$ и длительностью импульса $1.3 \mathrm{~ns}$ он составляет $24.6 \mathrm{~J} / \mathrm{cm}^{2}$ ) [4,6]. На сегодняшний день в кристаллах LBO были получены 700 и $160 \mathrm{~W}$ выходной мощности на длинах волн второй $(532 \mathrm{~nm})$ и третьей $(355 \mathrm{~nm})$ гармоник излучения иттербиевого лазера с эффективностями 70\% и $30 \%$ соответственно [7].

Несмотря на многообразие исследований различных свойств кристаллов LBO, влияние ионной проводимости на их оптические свойства на сегодняшний день практически не изучено. Известно, что для кристаллов LBO характерна ярко выраженная анизотропная ионная проводимость, обусловленная движением ионов лития $\mathrm{Li}^{+}$по свободным вакансиям [8,9]. Наибольшая ионная проводимость LBO наблюдается вдоль кристаллографической оси $c$, ее величина сильно зависит от 
температуры и частоты внешнего электрического поля. Температурная зависимость ионной проводимости $\sigma$ описывается феноменологическим законом Аррениуса:

$$
\sigma(T)=\frac{A}{T} \exp \left(-\frac{E_{a}}{k_{B} T}\right),
$$

где $T$ - температура образца, $E_{a}$ - энергия активации подвижных ионов, $k_{B}$ - постоянная Больцмана, $A-$ некоторый не зависящий от температуры множитель, определяемый концентрацией носителей заряда, расстоянием между вакансиями, частотой ионных прыжков. Зависимость ионной проводимости от частоты $f$ внешнего электрического поля определяется мнимой частью диэлектрической проницаемости $\varepsilon^{\prime \prime}$. Связь между $\sigma$ и $f$ описывается выражением [11]

$$
\sigma(f)=\sigma(0)+f \varepsilon_{0} \varepsilon^{\prime \prime}(f),
$$

где $\sigma(0)$ - проводимость при постоянном электрическом поле, $\varepsilon_{0}$ - диэлектрическая проницаемость вакуума.

В работе [9] для кристаллов LBO было получено, что при температуре $373 \mathrm{~K}$ и частоте внешнего электрического поля $50 \mathrm{kHz}$ значение проводимости вдоль оси $c$ составляет $\sigma_{c} \approx 10^{-6}(\mathrm{Ohm} \cdot \mathrm{cm})^{-1}$. Это значение в три раза больше величины ионной проводимости вдоль оси $a$, которая составляет $\sigma_{a} \approx 3 \cdot 10^{-7}(\mathrm{Ohm} \cdot \mathrm{cm})^{-1}$.

В работах $[11,12]$, были выдвинуты гипотезы о влиянии ионной проводимости на оптические свойства кристалла LBO, такие как порог оптического разрушения и коэффициент оптического поглощения. В работе [11] для трех длин волн импульсного излучения (1064, 532 и $355 \mathrm{~nm}$ ) наблюдалась ярко выраженная анизотропия порога оптического разрушения кристалла LBO в зависимости от направления поляризации. Причем на длине волны $1064 \mathrm{~nm}$ значение пороговой интенсивности оптического разрушения для поляризации излучения, параллельной оси $c$ кристалла более чем в три раза, превосходило значение порога для ортогональной поляризации. Авторами работы было сделано предположение, что в первом случае подвижные ионы лития способствуют уменьшению времени жизни формируемых центров окраски, что приводит к увеличению порога оптического разрушения кристалла LBO.

В работе [12] исследовались оптические свойства двух типов кристаллов LBO с ионной проводимостью (LBO1) и с подавленной ионной проводимостью (LBO-2). Было показано, что время начала деградации при взаимодействии с импульсным излучением на длине волны $266 \mathrm{~nm}$ для кристаллов LBO-2 примерно в два раза превосходит значение, характерное для LBO-1. Однако на длине волны $1064 \mathrm{~nm}$ коэффициенты оптического поглощения кристалла LBO-2, измеренные для ортогональных поляризаций лазерного излучения, отличаются в 4 раза. В случае кристаллов LBO-1 значения коэффициентов оптического поглощения для двух ортогональных направлений поляризации излучения практически совпадают. Также в этой работе было показано влияние ионной проводимости на форму линии пьезоэлектрических резонансов, возбуждаемых в кристаллах внешним радиочастотным электрическим полем. В кристаллах с подавленной ионной проводимостью добротность пьезоэлектрических резонансов не изменялась при разогреве. В кристаллах, где ионная проводимость не была подавлена, наблюдалось экспоненциальное уширение формы линии резонансов вплоть до их полного исчезновения. Предположительно, это обусловлено ростом величины ионной проводимости с температурой. Зависимость ширины линии $W$ пьезоэлектрического резонанса от температуры в этом случае хорошо описывается выражением [12]:

$$
W(T)=W_{0}+B \sigma(T),
$$

где $W_{0}$ - ширина линии резонанса при низких температурах $(T \sim 100 \mathrm{~K}), B$ - коэффициент пропорциональности, не зависящий от температуры.

Целью настоящей работы являлось исследование влияния ионной проводимости нелинейно-оптических кристаллов LBO на коэффициент оптического поглощения при воздействии непрерывного излучения высокой интенсивности методом пьезорезонансной лазерной калориметрии (ПРЛК). Для этого были предложены эксперименты по определению зависимости коэффициента оптического поглощения и коэффициента теплообмена кристалла трибората лития от интенсивности непрерывного одномодового неполяризованного лазерного излучения на длине волны $1070 \mathrm{~nm}$ в диапазоне от 10 до $100 \mathrm{MW} / \mathrm{cm}^{2}$. Стоит отметить, что для импульсного излучения малой и средней мощности оптическое поглощение кристаллов LBO на данной длине волны было исследовано ранее в работах [12]. Особенностью настоящей работы является использование непрерывного лазерного излучения с высокой мощностью, при которой и величина разогрева, и значение ионной проводимости в исследуемых кристаллах LBO могут значительно возрастать.

\section{Теоретическая часть}

Существуют различные методики измерения коэффициентов оптического поглощения диэлектрических материалов [13]. Международным стандартизованным методом измерения малых коэффициентов оптического поглощения является лазерная калориметрия [14]. В ее основе лежит поиск соответствия экспериментально измеренной кинетики разогрева исследуемого образца лазерным излучением с решением нестационарного уравнения теплопроводности

$$
\rho c_{s p} \frac{\partial T}{\partial t}=\nabla(\kappa \nabla T)+q,
$$

где $\rho-$ плотность, $c_{s p}-$ удельная теплоемкость, $\kappa-$ коэффициент теплопроводности (параметры образца), 
$T$ - температура, $q-$ функция, описывающая распределение источников тепла.

При решении уравнения, как правило, применяют граничные условия третьего рода (задача Робена), также известные как закон Ньютона-Рихмана [15]. На практике часто используют упрощенную модель разогрева, рассматривая уравнение теплового баланса [15]. В этом случае при воздействии лазерного излучения изменение температуры кристалла $\Delta T_{c}$ может быть описано следующим образом:

$$
\Delta T_{c}(t)= \begin{cases}0, & t \leq t_{1}, \\ \frac{\alpha l P}{\gamma m c_{s p}}\left(1-e^{-\gamma\left(t-t_{1}\right)}\right), & t_{1}<t<t_{2}, \\ \frac{\alpha l P}{\gamma m c_{s p}}\left(e^{-\gamma\left(t-t_{2}\right)}-e^{-\gamma\left(t-t_{1}\right)}\right), & t \geq t_{2},\end{cases}
$$

где $\alpha-$ коэффициент оптического поглощения, $P$ мощность лазерного излучения, $m$ - масса образца, $l$ - длина образца, $\gamma=\left(h^{T} S\right) /\left(m c_{s p}\right)-$ коэффициент температурных потерь, $h^{T}$ - коэффициент теплообмена, $S$ - площадь поверхности кристалла, $t_{1}$ - время начала облучения, $t_{2}$ - время завершения облучения. Измерив экспериментально кинетику разогрева исследуемого кристалла лазерным излучением и аппроксимировав ее экспоненциальной функцией (5), можно определить коэффициенты оптического поглощения $\alpha$ и теплообмена $h^{T}$.

В методе лазерной калориметрии температура образца обычно измеряется с помощью внешних термодатчиков (термопара, терморезистор идр.). Основной недостаток такого подхода заключается в том, что рассеянное излучение приводит к дополнительному нагреву термодатчика, который в случае воздействия излучения высокой интенсивности может быть очень существенным.

Метод ПРЛК основан на использовании концепции эквивалентной температуры, подробно описанной в работах $[16,17]$. При воздействии лазерного излучения мощностью $P$ эквивалентная температура $\theta_{e q}$ нелинейнооптических кристаллов, обладающих пьезоэлектрическими свойствами, определяется напрямую по измерению индуцированного сдвига частот $\Delta R f_{n}(P)$ пьезоэлектрических резонансов, калиброванных по температуре в условиях однородного разогрева:

$$
\Delta \theta_{e q}(P)=\frac{\Delta R f_{n}(P)}{K_{n}^{p r t}},
$$

где пьезорезонансный термический коэффициент $K_{n}^{p r t}=d R f_{n} / d T$ данной моды находится на основе предварительной калибровки зависимостей резонансных частот от температуры при однородном разогреве, которые в первом приближении линейны.

Таким образом, измерение кинетики резонансной частоты выбранной моды при разогреве кристалла лазерным излучением заданной интенсивности определяет

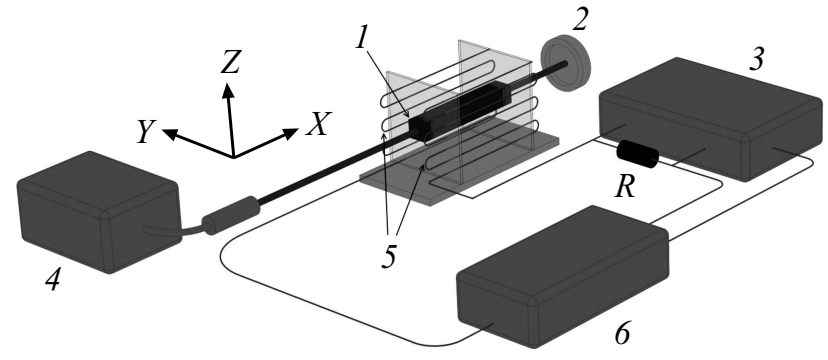

Рис. 1. Блок-схема экспериментальной установки для измерения малых коэффициентов оптического поглощения нелинейно-оптических кристаллов методом ПРЛК: 1 - исследуемый кристалл LBO, 2 - измеритель мощности оптического излучения, 3 - синхронный детектор, 4 - источник лазерного излучения, 5 - металлические электроды, $6-$ радиочастотный генератор.

кинетику эквивалентной температуры в соответствии с выражением (6). Коэффициенты оптического поглощения $\alpha$ и теплообмена $h^{T}$ определяются из решения системы (5) заменой термодинамической температуры $T_{c}$ на эквивалентную $\theta_{e q}$.

На практике при малых значениях коэффициентов оптического поглощения вполне достаточно использовать начальный участок $(5-10 \mathrm{~s})$ кинетики разогрева кристалла, так как, исходя из вида решения (5), на начальном участке экспоненту можно разложить в ряд Тейлора с учетом малости экспоненциального показателя [18]. В этом случае температура кристалла будет линейно зависеть от времени с наклоном, прямо пропорциональным коэффициенту оптического поглощения:

$$
\Delta T_{c}(t)=\frac{\alpha l P}{m c_{s p}}\left(t-t_{1}\right) .
$$

После замены термодинамической температуры $T_{c}$ на эквивалентную $\theta_{e q}$, определяемую по формуле (6), коэффициент оптического поглощения определяется из выражения:

$$
\alpha=\frac{m c_{s p} k_{n}}{l P K_{n}^{p r t}},
$$

где $k_{n}=d R f_{n} / d t$ соответствует углу наклона начального участка измеренной кинетики резонансной частоты.

\section{Экспериментальная установка}

Упрощенная блок-схема экспериментальной установки для измерения малых коэффициентов оптического поглощения методом ПРЛК представлена на рис. 1.

В настоящей работе исследовался образец кристалла LBO в форме прямоугольного параллелепипеда с размерами $3 \times 3.3 \times 20 \mathrm{~mm}$. Кристалл вырезан по кристаллографическим осям (кристаллографическая ось $c$ направлена вдоль оси $Y$, размер кристалла вдоль оси $Y$ составляет $3.3 \mathrm{~mm}$ ). Исследуемый образец помещался в конденсатор, образованный электродами из медной 
проволоки диаметром $200 \mu \mathrm{m}$; проволока продевалась через отверстия в двух вертикальных тонких пластинах, изготовленных из плавленого кварца. Такая конфигурация позволяет минимизировать разогрев окружающего кристалл пространства, обусловленный поглощением рассеянного излучения. Напряжение с радиочастотного (РЧ) генератора подавалось в цепь, содержащую последовательно соединенные конденсатор с кристаллом и нагрузочное сопротивление $R$. Напряжение на нагрузочном резисторе измерялось синхронным детектором. На опорный вход синхронного детектора с выхода генератора подавался РЧ сигнал той же частоты. При фиксированной амплитуде напряжения РЧ генератора для каждого значения частоты $f$ синхронным детектором измерялась амплитуда $\left|U_{R}\right|$ и фаза $\varphi$ напряжения на нагрузочном сопротивлении $R$.

Источником непрерывного неполяризованного излучения является волоконный одномодовый иттербиевый лазер с длиной волны излучения $\lambda=1070 \mathrm{~nm}$, выходной мощностью излучения до $500 \mathrm{~W}$ и параметром качества пучка $M^{2}=1.05$. Лазерный пучок фокусировался линзой в центр кристалла LBO. Диаметр перетяжки составлял $35 \mu \mathrm{m}$, диаметр пучка на торцах кристалла $0.43 \mathrm{~mm}$. Максимальная интенсивность излучения в перетяжке при максимальной мощности лазерного излучения порядка $100 \mathrm{MW} / \mathrm{cm}^{2}$.

\section{Экспериментальные результаты}

В ходе эксперимента в диапазоне частот от 0.5 до $1.5 \mathrm{MHz}$ была измерена спектральная зависимость отклика (по амплитуде и фазе напряжения на нагрузочном резисторе $R$ ) кристалла LBO на воздействие внешнего радиочастотного поля. Для определения коэффициента оптического поглощения $\alpha$ методом ПРЛК был выбран резонанс на частоте $1074.5 \mathrm{kHz}$ (при температуре $\left.T_{1}=25^{\circ} \mathrm{C}\right)$. Резонансной частотой $R f$ можно считать частоту, при которой достигается минимальное значение фазы измеряемого сигнала.

Температурная калибровка частоты выбранного пьезоэлектрического резонанса производилась в условиях однородного разогрева. Конденсатор с кристаллом был помещен в термостат. Температура образца изменялась в диапазоне от 25 до $50^{\circ} \mathrm{C}$. На рис. 2 приведены спектральные зависимости фазы напряжения, измеренного вблизи пьезоэлектрического резонанса при температурах $T_{1}=25^{\circ} \mathrm{C}$ и $T_{2}=35^{\circ} \mathrm{C}$. Видно, что при разогреве резонансная линия существенно уширяется, что, согласно результатам работы [12] и приведенному выражению (3), обусловлено ростом величины ионной проводимости кристалла LBO при разогреве. На рис. 3 представлена зависимость сдвига резонансной частоты от температуры при однородном разогреве кристалла. Из линейной аппроксимации данной зависимости с использованием формулы (6) был определен пьезо-

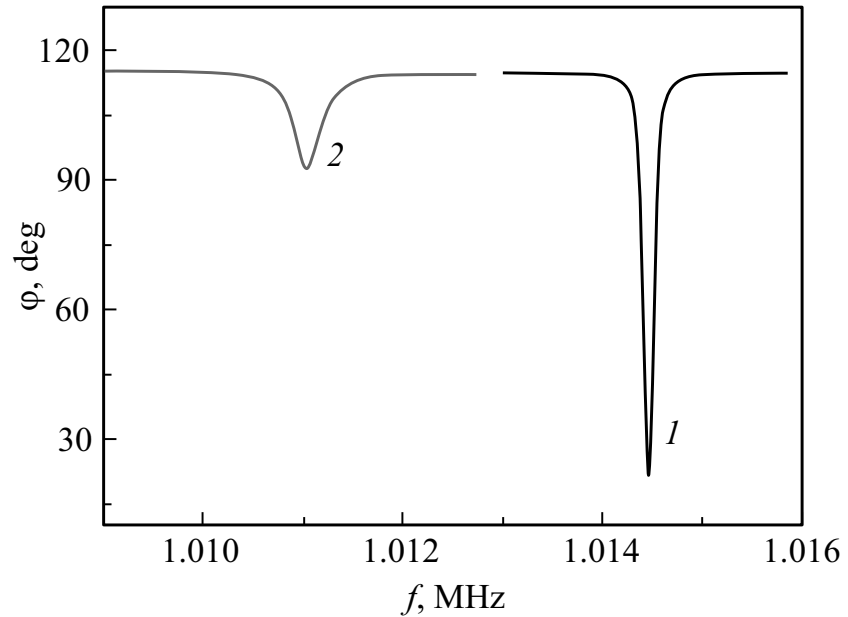

Рис. 2. Спектральная зависимость фазы измеряемого напряжения вблизи частоты пьезоэлектрического резонанса кристалла LBO при разных температурах: $1-25^{\circ} \mathrm{C}, 2-35^{\circ} \mathrm{C}$.

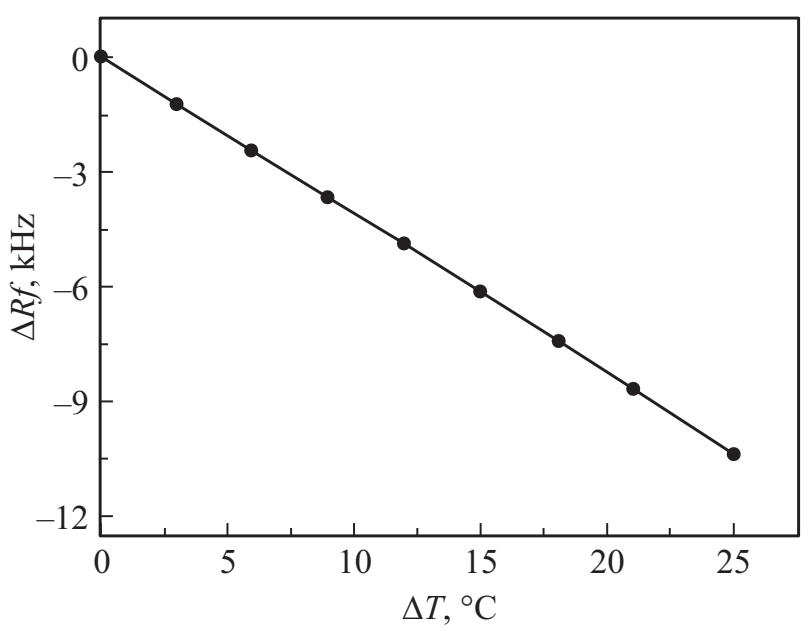

Рис. 3. Зависимость сдвига частоты пьезоэлектрического резонанса кристалла LBO от температуры при однородном разогреве.

резонансный термический коэффициент данной моды $K^{p r t}=(0.42 \pm 0.02) \mathrm{kHz} /{ }^{\circ} \mathrm{C}$.

Была измерена величина разогрева кристалла LBO в зависимости от интенсивности лазерного излучения. Максимальное изменение эквивалентной температуры кристалла $\Delta \theta_{e q}^{\max }$ для каждого значения интенсивности лазерного излучения фиксировалось при достижении стационарного режима. Полученная зависимость представлена на рис. 4. Оптотермический коэффициент, рассчитанный из линейной аппроксимации, составил (при пересчете в единицы $\left.{ }^{\circ} \mathrm{C} / \mathrm{kW}\right) \beta=(26 \pm 4)^{\circ} \mathrm{C} / \mathrm{kW}$.

Коэффициент теплообмена исследуемого кристалла определялся на основе аппроксимации экспериментально измеренной кинетики разогрева кристалла по формуле (5), в которой термодинамическая температура была заменена эквивалентной. Зависимость величины коэф- 
фициента теплообмена исследуемого кристалла LBO от интенсивности лазерного излучения представлена на рис. 5. Усредненное значение коэффициента теплообмена составило $h^{T}=(33 \pm 8) \mathrm{W} /\left(\mathrm{m}^{2}{ }^{\circ} \mathrm{C}\right)$.

Коэффициент оптического поглощения кристалла LBO на длине волны $\lambda=1070 \mathrm{~nm}$ был измерен в диапазоне интенсивностей лазерного излучения $10-100 \mathrm{MW} / \mathrm{cm}^{2}$. На рис. 6 приведены начальные участки кинетики резонансной частоты, измеренные при воздействии лазерного излучения с интенсивностью $I_{1}=10 \mathrm{MW} / \mathrm{cm}^{2}, I_{2}=20 \mathrm{MW} / \mathrm{cm}^{2}$ и $I_{3}=100 \mathrm{MW} / \mathrm{cm}^{2}$ соответственно.

Для всех значений интенсивности лазерного излучения коэффициент оптического поглощения $\alpha$ определялся с использованием формулы (8). Результаты расчетов представлены на рис. 7. Величина коэффициента оптического поглощения кристалла LBO на длине волны

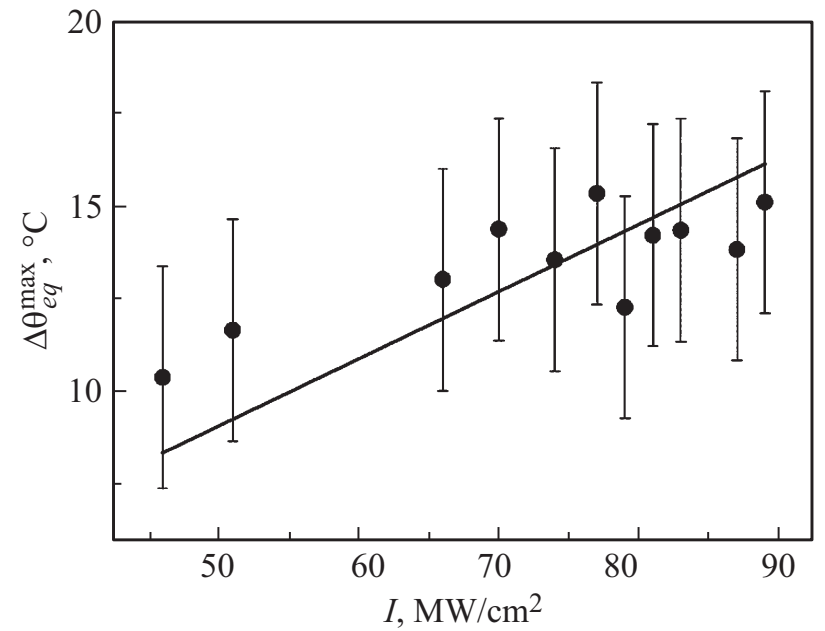

Рис. 4. Зависимость изменения эквивалентной температуры кристалла LBO от интенсивности лазерного излучения на длине волны $1070 \mathrm{~nm}$.

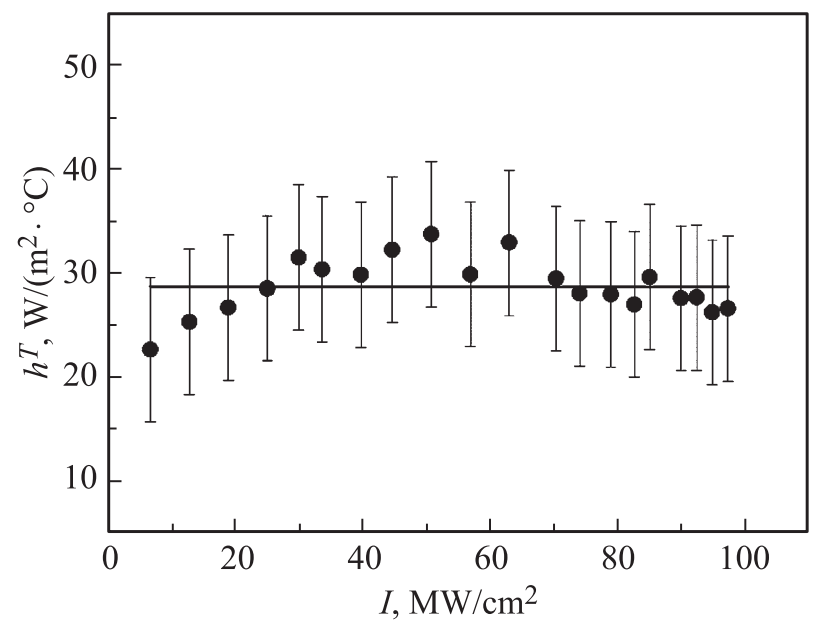

Рис. 5. Зависимость коэффициента теплообмена кристалла LBO от интенсивности лазерного излучения на длине волны $1070 \mathrm{~nm}$.

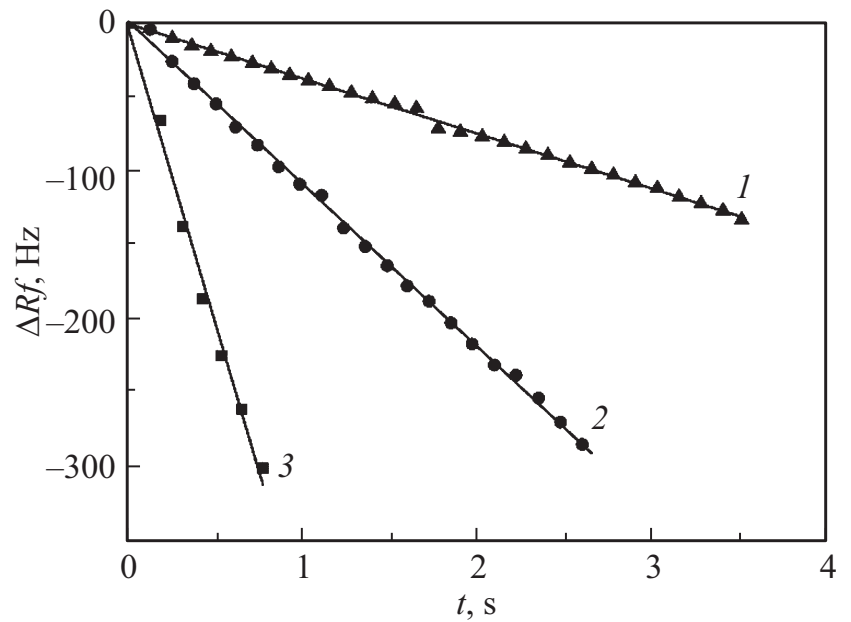

Рис. 6. Начальные участки кинетики резонансной частоты кристалла LBO при воздействии лазерного излучения различных интенсивностей: $1-10,2-20,3-100 \mathrm{MW} / \mathrm{cm}^{2}$.

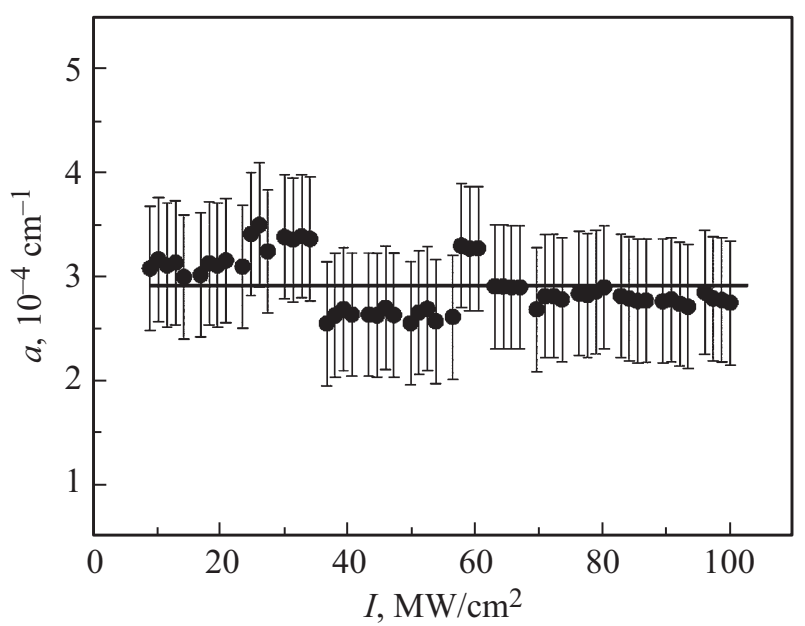

Рис. 7. Зависимость коэффициента оптического поглощения кристалла LBO от интенсивности лазерного излучения на длине волны $1070 \mathrm{~nm}$.

$\lambda=1070 \mathrm{~nm}$ не зависит от интенсивности лазерного излучения в данном диапазоне, среднее значение составило $\alpha=(2.9 \pm 0.7) \cdot 10^{-4} \mathrm{~cm}^{-1}$.

\section{Выводы}

Полученные в настоящей работе результаты показывают, что коэффициент оптического поглощения кристалла LBO при воздействии непрерывного лазерного излучения на длине волны $1070 \mathrm{~nm}$ в диапазоне интенсивностей $10-100 \mathrm{MW} / \mathrm{cm}^{2}$ остается постоянным и имеет значение $\alpha=(2.9 \pm 0.7) \cdot 10^{-4} \mathrm{~cm}^{-1}$. Ранее в радиочастотном диапазоне было обнаружено существенное влияние ионной проводимости LBO на форму линии пьезоэлектрических резонансов при разогреве. Однако влияние ионной проводимости на коэффициент 
оптического поглощения кристалла LBO в диапазоне интенсивностей $10-100 \mathrm{MW} / \mathrm{cm}^{2}$ непрерывного лазерного излучения на длине волны $1070 \mathrm{~nm}$ не было выявлено. Было продемонстрировано, что метод ПРКЛ позволяет корректно измерять коэффициенты оптического поглощения нелинейно-оптических кристаллов в широком диапазоне интенсивностей лазерного излучения.

\section{Финансирование работы}

Работа выполнена в рамках темы госзадания.

\section{Конфликт интересов}

Авторы заявляют, что у них нет конфликта интересов.

\section{Список литературы}

[1] Shiner B. // Opt. Soc. Am. CLEO: Applications and Technology. 2013. AF2J.1.

[2] Ishida Y., Yajima T. // Opt. Commun. 1987. V. 62 (3). P. $197-$ 200.

[3] Chen C. et al. // J. Opt. Soc. Am. 1989 V. 6 (4). P. 616-621.

[4] Nikogosyan D.N. Nonlinear Optical Crystals: a Complete Curvey. NY: Springer Science \& Business Media, 2006.

[5] Vershinin O.I., Konyashkin A.V., Ryabushkin O.A. // Opt. Lett. 2018. V. 43 (1). P. 58-61.

[6] Блистанов А.А. Кристаллы квантовой и нелинейной оптики. М.: МИСИС, 2000.

[7] Avdokhin A. et al. // Proc. SPIE Nonlinear Freq. Gener. Convers.: Mater. Devices, Appl. XIV. 2015. V. 9347. P. 934704.

[8] Радаев С.Ф., Сорокин Н.И., Симонов В.И. // ФТТ. 1991. T. 33 (12). C. $3597-3600$

[9] Kannan C.V., Ganesamoorthy S., Rajesh D., Jayavel R., Kimura H. // Solid State Commun. 2005. V. 136 (4). P. 215219.

[10] Kim J.W., Yoon C.S., Gallagher H.G. // Appl. Phys. Lett. 1997. V. 71 (22). P. 3212-3214.

[11] Wagner F.R., Hildenbrand A., Natoli J.Y., Commandré M. // Optics Express. 2010. V. 18 (26). P. 26791-26798.

[12] Nikitin D.G., Pigarev A.V., Konyashkin A.V., Ryabushkin O.A. // Opt. Lett. 2018. V. 43 (19). P. 4843-4846.

[13] Hordvik A. // Appl. Opt. 1977. V. 16 (11). P. 2827-2833.

[14] ISO 11551:2003 Test Method for Absorptance of Optical Laser Components.

[15] Willamowski U., Ristau D., Welsch E. // Appl. Opt. 1998. V. 37 (36). P. 8362-8370.

[16] Рябушкин О.А., Мясников Д.В. // Квант. электрон. 2012. T. 42 (6). C. 539-544.

[17] Ryabushkin O.A., Myasnikov D.V., Konyashkin A.V., Tyrtyshnyy V.A. // J. Eur. Opt. Soc. Rapid Publ. 2011. V. 6. P. 11032.

[18] Pigarev A.V., Konyashkin A.V., Ryabushkin O.A. // Proc. SPIE Nonlinear Opt. Appl. IV. 2016. V. 9894. P. 98941. 